\section{Tumortherapiefelder mit längerem Überleben bei Glioblastompatienten assoziiert}

\author{
Wird die Erhaltungsbehandlung von Glioblastompatienten mit \\ Tumortherapiefeldern unterstützt, verlängert sich die Zeit bis zur \\ Progression ebenso wie das Gesamtüberleben.
}

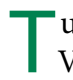
umortherapiefelder (TTF) sind ein Verfahren, das in die Mitose eingreift. Mit einer Frequenz von $200 \mathrm{kHz}$ erzeugte elektrische Wechselfelder sollen dabei am Tumor mit einer Feldstärke von 1-2 $\mathrm{V} / \mathrm{cm}$ wirken. Das Ziel ist es, die Bildung des Spindelapparates während der Metaphase der Zellteilung zu unterbrechen und eine dielektrophoretische Bewegung polarer Moleküle zu verursachen. Diese Prozesse sollen eine Apoptose induzieren. Die Applikation der Wechselfelder erfolgt über 4 äußere Elektroden, die an der kahlrasierten Kopfhaut angebracht werden. Gespeist wird das System aus einer im Rucksack mitzuführenden Batterie. Die tägliche Anwendungsdauer beträgt mehr als 18 Stunden.

Roger Stupp, Zürich, Schweiz, hat TTF zusammen mit einem internationalen Forscherteam in einer Phase-III-Studie bei Patienten getestet, die an einem supratentorialen Glioblastom erkrankt waren und noch kein Rezidiv erlitten hatten. Die Patienten hatten sich einer Operation und einer Radiochemotherapie unterzogen. Nun befanden sie sich in der Erhaltungstherapie-Phase, in der sie über 6-12 Monate alle 4 Wochen 5 Tage lang Temozolomid erhalten sollten (150-200 mg/ $\mathrm{m}^{2} / \mathrm{d}$ ). Von Juli 2009 bis November 2014 wurden 695 Patienten 2:1 auf die Behandlung mit Temozolomid plus TTF oder Temozolomid allein randomisiert.

Stupp und Kollegen publizierten nun die Ergebnisse einer Interimsanalyse, die auf den Daten von 315 Patienten beruht - 210 hatten Temozolomid plus TTF erhalten, 105 nur Temozolomid. Das Follow-up dauerte im Median 38 Monate (18-60 Monate). Bei Patienten, die zusätzlich TTF erhalten hatten, war das progressionsfreie Überleben (PFS) in der Intention-to-treat(ITT)-Analyse länger als bei Patienten mit Temozolomid-Monotherapie (7,1 vs. 4,0 Monate). Auch das mediane Gesamtüberleben (OS) war unter Temozolomid plus TTF länger (20,5 vs. 15,6 Monate). Die Berechnung erfolgte hier gemäß Protokoll, eine zusätzliche ITT-Analyse ergab aber keine wesentlichen Unterschiede.

Fazit: Die unterstützende Behandlung mit TTF war bei den Glioblastompatienten mit signifikant längeren Überlebenszeiten assoziiert. Wie viel die elektrischen Wechselfelder zu diesem Erfolg beigetragen haben - der sogar zum Studienabbruch führte, um die Kontrollgruppe ebenfalls mit TTF versorgen zu können -, ist dennoch nicht geklärt. Robert Bublak

Stupp R et al. Maintenance Therapy With TumorTreating Fields Plus Temozolomide vs Temozolomide Alone for Glioblastoma - A Randomized Clinical Trial. JAMA. 2015;314(23):2535-43.

Die Daten der Studie von Roger Stupp und Kollegen sind seit mehr als 1 Jahr bekannt. Bisher ist der Einsatz der Therapie aber in Deutschland - und auch in anderen Ländern Europas und in Israel - sehr limitiert. In den meisten Zentren werden Patienten zwar über die Behandlung informiert, die zusätzlich zur Erhaltungstherapie mit Temozolomid eingesetzt werden kann, es erfolgen jedoch seit Abschluss der Studie nur vereinzelt Behandlungen. Ein Grund für eine insgesamt recht geringe Akzeptanz ist wohl vor allem die Zurückhaltung der Patienten gegenüber der langfristig durch Kopfhaarentfernung und Mitführen eines Geräts belastenden Therapieform. Eine Progressionstherapiestudie, die einige Jahre früher abgeschlossen worden war, hatte keinen relevanten Effekt ergeben [Stupp R et al. Eur J Cancer. 2012;48(14): 2192-202].

Die behandelnden Ärzte sind noch nicht flächendeckend mit dem Gerät und der Organisation der Kostenseite vertraut. Womöglich bedarf es auch einiger Gewöhnung, dass ein Techniker der Firma durch die erforderlichen, regelmäßigen Hausbesuche Teil des Therapieteams wird. Bei den Experten

\section{Kommentar von Prof. Wick:}

besteht wegen der ungewöhnlichen Studienpopulation Unsicherheit, ob die Studiendaten generalisierbar sind und wie geeignete Patienten ausgewählt werden können. Im Mittel wurden die Patienten erst 3,8 Monate nach Diagnose in die Studie eingeschlossen. Zu dieser Zeit haben etwa 30\% der Patienten mit einer Glioblastom-Standardtherapie bereits einen Progress entwickelt. Offenbar spielt die recht hohe Zahl von Patienten, die den Studienendpunkt zum Zeitpunkt der Auswertung noch gar nicht erreicht haben, keine entscheidende Rolle. Ein möglicher Einfluss der zusätzlichen supportiven Maßnahmen durch die regelmäßigen Hausbesuche auf das PFS und auch das OS lässt sich jedoch nicht ausschließen. Bei Patienten mit fortgeschrittenem Lungenkrebs wurde in einer Studie durch gezielte, frühzeitige palliative Therapie ein relevanter Überlebensvorteil erreicht [Temel JS et al. N Engl J Med. 2010;363(8):733-42].

Der primäre Endpunkt der Studie, das PFS, ist wegen der nicht auszuschließenden Ein-

\section{"Für alle Skeptiker sollte eine Studie mit Scheintherapie in der Kontrollgruppe erfolgen."}

flüsse der Radiochemotherapie auf die Magnetresonanztomografie(MRT)-Ergebnisse (sogenannte Pseudoprogression) nicht unumstritten und in anderen Studien heftig kritisiert worden. Als Unterstützung sollten die Effekte auf das OS trotz aktuell noch vieler zensierter Patienten angesehen werden. Maßstab sollte hier die ITT-Population sein, in der die Effekte weiterhin vorhanden, jedoch weniger stark zu sein scheinen.

Für die negative Einschätzung einer Progressionstherapie ergibt die aktuelle Studie im übrigen keine neuen Gesichtspunkte. TTF sind aber eine Option für die Primärtherapie und sollten in einer bestätigenden Studie z. B. in einer komplementären Population -, die am besten mit Scheintherapie kontrolliert durchgeführt wird, für alle Skeptiker nochmals sorgfältig untersucht werden.

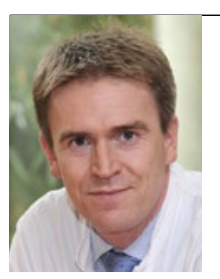

Prof. Dr. med. Wolfgang Wick Neurologische Klinik und NCT der Universitätsklinik Heidelberg wolfgang.wick@med. uni-heidelberg.de 